\title{
Cultural Competence in Medical Education: Aligning the Formal, Informal and Hidden Curricula
}

\section{Authors}

David Paul MBBS PhD, Shaun Ewen B.App.Sc. (Physio) MMIL DEd, Rhys Jones $\mathrm{MBChB} \mathrm{MPH}$

Professor Paul is Associate Dean, Aboriginal Health, School of Medicine (Fremantle), University of Notre Dame Australia, Fremantle, Western Australia, Australia.

(Formerly, Deputy Director, Centre for Aboriginal Medical and Dental Health, University of Western Australia)

Associate Professor Ewen is Deputy Director, Centre for Health and Society, The University of Melbourne, Melbourne, Victoria, Australia.

Dr Jones is Senior Lecturer, Te Kupenga Hauora Māori, University of Auckland, Auckland, New Zealand.

Correspondence should be addressed to Professor David Paul, School of Medicine, Fremantle, University of Notre Dame, PO Box 1225, Fremantle, Western Australia 6959; telephone: +61 8 94330525; fax: +61 8 94330250; e-mail:

david.paul@nd.edu.au 


\begin{abstract}
The concept of cultural competence has become reified by inclusion as an accreditation standard in the US and Canada, in New Zealand it is demanded through an Act of Parliament, and it pervades discussion in Australian medical education discourse. However, there is evidence that medical graduates feel poorly prepared to deliver cross-cultural care (Weissman, Betancourt et al. 2005) and many commentators have questioned the effectiveness of cultural competence curricula. In this paper we apply Hafferty's taxonomy of curricula, the formal, informal and hidden curriculum (Hafferty 1998), to cultural competence. Using an example across each of these curricular domains, we highlight the need for curricular congruence to support cultural competence development among learners. We argue that much of the focus on cultural competence has been in the realm of formal curricula, with existing informal and hidden curricula which may be at odds with the formal curriculum. The focus of the formal, informal and hidden curriculum, we contend, should be to address disparities in health care outcomes. In conclusion, we suggest that without congruence between formal, informal and hidden curricula, approaches to addressing disparity in health care outcomes in medical education may continue to represent reform without change.
\end{abstract}

\title{
Key Words
}

Medical Education, Cultural Competence, Health Disparity, Hidden Curriculum, Minorities. 


\section{Introduction}

As medical educators grapple with how to best prepare graduates to contribute to the health and wellbeing of all populations, including historically underserved and under represented minorities, cultural competence has come to the fore (Perloff, Bonder et al. 2006; Saha, Beach et al. 2008). Cultural competence is now evident in accreditation requirements in North America, Australia and New Zealand (New Zealand Government 2003; Australian Medical Council 2007; Liaison Committee on Medical Education 2012). Across a similar time frame, recognition of the influences of not only the formal, but also the informal and hidden curricula on the learner has increased.

Much of the focus on cultural competence education to date has been in the formal curriculum, with little explicit analysis of the impact of other aspects of the learning environment on cultural competence development. In this paper we apply Hafferty's taxonomy of curricula, Formal, Informal and Hidden, to cultural competence education (Hafferty 1998). This paper describes the evolution of cultural competence curricula in a medical education context, exposing the sometimes-divergent aims of the formal curriculum. Then follows a description of the influence of the informal curriculum on cultural competence, using the health workforce demographic as an example. Finally, the hidden curriculum's relationship with cultural competence is discussed, with particular focus on the term 'cultural competence' as institutional slang. 


\section{Cultural competence}

The notion of cultural competence was originally developed to provide a philosophical framework as well as practical ideas that would help to improve service delivery to children of "color" who were "severely emotionally disturbed" (Cross, Bazron et al. 1989 p. 7), and as a tool to help overcome cultural differences between the assumed "non-colored" clinician, and the "colored" child. Within this framework cultural competence was described as a:

... set of congruent behaviors, attitudes, and policies that come together in a system, agency, or amongst professionals and enables that system, agency, or those professionals to work effectively in cross-cultural situations (Cross, Bazron et al. 1989 p. iv).

Our interpretation is that Cross' definition essentializes and problematizes "children of color", constructing "colored" "race" as static, Other, and different from the presumably "non-colored" clinician. This conceptualization has influenced much cultural competence education in the intervening period, with curricula focusing on learning about the 'cultures' of different (predominantly minority ethnic) groups (Taylor 2003; Weissman, Betancourt et al. 2005; Gregg and Saha 2006; Wear, Kumagai et al. 2012)

More recently there has been a shift such that the main driver of the:

... cultural competence movement ... has been the demonstration of and publicity surrounding widespread racial and ethnic disparities in healthcare (Saha, Beach et al. 2008 p. 1278).

There is increasing evidence of substantial disparities in health care outcomes and interventions for people who are from ethnic minority groups (Smedley, Stith et al. 2003; Coory and Walsh 2005; Harris, Robson et al. 2007), with the emergent challenge to bring the same rigor to research which examines inequity in other 
contexts, such as age, gender or sexuality (Kumas-Tan, Beagan et al. 2007). The increased discussion of cultural competence in the medical education literature as well as in medical education accreditation requirements (Australian Medical Council 2007; Liaison Committee on Medical Education 2012) has been so pervasive that the term has:

... become a byword endowed with almost religious significance, a panacea for the multiple and interwoven problems in health care communication (Perloff, Bonder et al. 2006 p. 835).

The pervasive, and sometimes confusing and divergent aims of cultural competence education should not be allowed to cloud educator's minds, as we strive to ensure we graduate the best clinicians we can.

\section{Formal cultural competence curricula}

Given potentially divergent reasons for investing in cultural competence curricula, it is critical for medical educators to clearly identify the purposes for which they are developing and implementing such curricula. Different aims and rationales require different approaches, pedagogies, and methods of evaluation. An approach designed to understand a static Other will be different from an approach designed to facilitate students' awareness of, and ability to respond to, disparity in health care outcomes. Addressing health care disparities should be the primary reason for inclusion of cultural competence curricula for health care professionals. The literature on disparities in health care identifies a complex interaction of contributing factors including health system, health professional and patient factors (Smedley, Stith et al. 2003). The health professional factors identified in this literature predominantly revolve around psychological processes including unconscious bias and stereotypes as well as differences in clinician-patient communication (van Ryn and Fu 2003; Burgess, Fu et al. 2004; Klonoff 2009; Penner, Dovidio et al. 2010; Haider, Sexton et 
al. 2011). Educating health professionals to know more about the Other is therefore unlikely, in isolation, to address the underlying determinants of health care disparities. However, supporting health professionals to have greater self-awareness and critical consciousness is more clearly directed towards addressing the health professional factors identified in the literature (Kumagai and Lypson 2009). The implication is that formal curriculum initiatives aimed at addressing disparities in health care outcomes may be more appropriately directed to focus on:

- developing self reflexivity, including interventions to help learners identify and correct for unconscious bias and stereotyping (Kumas-Tan, Beagan et al. 2007; Pon 2009; Teal, Gill et al. 2012)

- understanding the historical and social interplay which contributes to the constructed nature of the Other, as it relates to the clinical context of patientcentered care. (Saha, Beach et al. 2008)

- communication skills, with a focus on the complexity of clinical interactions rather than simplistic or formulaic notions of communicating with the Other (Perloff, Bonder et al. 2006)

These curricular initiatives, designed, developed and locally implemented, are clearly linked to meeting the aims of cultural competence curricula, as they draw on evidence to address clinician contribution to disparity in health care outcomes.

\section{Informal cultural competence curricula challenges}

The informal curriculum consists of the interpersonal interactions between teachers and students, between students themselves, the conversations that occur in the corridor and café, possibly the influence of societal values, norms and popular culture discourses on those conversations, and therefore also the influence of role models on what, and how, students learn (Hafferty 1998). Given the socio-demographic representation of the health workforce, these informal discussions are likely to occur 
in the absence of underrepresented minorities, the very population groups who are most affected by disparity in health care outcomes. In an Australian context, for example, Indigenous Australians make up $0.2 \%$ of the health workforce (Australian Institute of Health and Welfare 2011), more than a ten-fold under-representation compared with population parity. It is the authors' experiences that most medical student learning related to Indigenous health is primarily constructed through case based learning examples, or as patient or public health data, but rarely as physician, colleague, or equal. Whilst this represents an extreme case in Western medical training, parallels can be drawn with US and Canadian student and health professional demographics in relation to under-represented minorities, with African Americans and Hispanics constituting 3 and $4 \%$ of medical faculty respectively, compared with 12.2 and $15.4 \%$ of the US population (Merchant and Omary 2010 p. 21).

The impact of the informal curriculum is highlighted in the experiences of Australian Indigenous students in medical school who reported:

... being affected by discrimination directed both at individual students and at Indigenous people as a group. Discrimination was described as being both overt and subtle. Participants commented that 'you can always sense it, but it's never out in the open (Garvey, Rolfe et al. 2009 p. 1053).

The Indigenous students described the informal curricula at the level of interpersonal interaction, which was experienced as discriminatory. One could imagine that the discriminatory inclinations persisted, and were possibly more overt as a result of being subject to less censorship, when no Indigenous students or faculty were present (Garvey, Rolfe et al. 2009), a most likely scenario given the workforce demographics. Students learn from role models and the concept of the informal curriculum 'allows us to better address and assess the importance of role models in the learning that takes place at all levels of medicine' (Hafferty 1998 p. 404). The proportional lack of ethnic 
diversity and representation within the medical workforce means that the majority demographic has a more significant influence over the informal curriculum. The influence of the informal curricula may reinforce notions of the Other as not 'belonging' in the medical profession. "They" are under-represented in number, in influence, and therefore "their" views and ways of working are not as accessible as role models, and thus have a lesser impact on student learning through the informal curricula. Ensuring equitable workforce representation is one strategy, however, if implemented in isolation it will not fully address this problem. In an Australian or New Zealand context, Indigenous representation at population parity will still constitute a minority within the health workforce. Hence the value of also implementing compulsory training programs for all faculty as a key strategy towards addressing the impact of workforce underrepresentation of some population groups. It also enhances the likelihood of congruence between the formal and informal curriculum, as the

... training and evaluation of faculty and clinicians on cultural diversity issues are very important since they serve as role models and their poor modelling may detract from work done in earlier years at medical school (Dogra, Reitmanova et al. 2009 p. 992).

In the absence of minority role models, the majority faculty must redouble its own efforts; to ensure their role modeling is reflexive in regards to issues of cultural competence. This could be achieved by utilizing continuing professional development opportunities for all faculty (including those primarily based in clinical sites) to help ensure that formal curricular initiatives are reinforced by the informal curriculum.

\section{Hidden curriculum and cultural competence}

In attempting to uncover the hidden curriculum, Hafferty suggested educators, 'pay particular attention to four areas: (1) policy development, (2) evaluation, (3) resource 
allocation, and (4) institutional “slang” or nomenclature' (Hafferty 1998 p.404). We propose that the terminology, "cultural competence" can be considered institutional slang, and will use this as an example of the hidden curriculum for this paper. Hafferty reminds us that the hidden curriculum "can be unearthed in the language used within organizations" (Hafferty 1998 p. 405). In other words, institutional slang, as an element of the hidden curriculum, can be used as a lens for critiquing the language of cultural competence. As a form of slang, it is most closely aligned to the definition of terminologic usage violations, which is "the facetious application of ... terminology in violation of accepted rules of usage" (Burson-Tolpin 1989 p. 286). The teaching of cultural competence is rarely, if ever, about the culture of the medical profession or the clinician herself (Wear, Kumagai et al. 2012), and in such a context is much more focused on the Other.

In many cases, "culture" becomes slang for "different". That difference is rarely described in relational terms; rather, the Other is identified as different, aberrant or abnormal (in contrast to an invisible, but implicitly understood, 'normal' dominant culture). This is compounded by a trend in medical education "which systematically tends to foster static and essentialist conceptions of "culture" as applied to patients" (Taylor 2003 p. 555). And thus, understanding the Aborigine, the Māori, the African American or the Hmong becomes the impossible aim of the learning exercise. The language of competence also lends itself to analysis as slang. The adoption of a competency-based approach to medical education is not confined to the issue of disparity in health care outcomes - it has become embedded into all facets of the education project. However the underpinning notion of a linear continuum from incompetence to competence is limited (Paul, Hill et al. 2012). That there is an achievable, acceptable standard that once achieved is all that the practitioner requires is problematic. The continuum of learning about oneself, one's profession, and the 
ever-diverse interactions with patients, lends itself to a lifelong learning process, rather than reaching an endpoint of competence. Critics of a competence approach posit that "competency is not what we want to use when trying to determine if someone is a good, or even an adequate, physician” (Brooks 2009 p. 91).

"Competence", as an endpoint to a learning outcome, should be considered an anathema to professionalism.

We would argue that a more complex, nuanced, "values based" approach that is taught consistently provides an alternative frame (Stern 1998). The object being not mastery of a finite body of knowledge, skills and attitudes that the notion of competency imbues, but rather:

... the continuous critical refinement and fostering of a type of thinking and knowing - a critical consciousness - of self, others, and the world (Kumagai and Lypson 2009 p. 783).

The challenge remains to implement professional development programs for health professional educators that will develop that reflexivity to enable the more ready embrace of such approaches. As such, an approach to addressing 'cultural competence' as slang would be to rename it. Here we can potentially step into a minefield of possibilities, however, in a global context, institutions could look to their own developed graduate attributes or outcomes, and focus on those which are relevant to disparity in health care outcomes. We suggest that these would include professionalism and reflexive and ethical practice. Further analysis could be undertaken linking graduate attributes or outcomes to how each institution understands the aims and practices related to cultural competence, and in light of the critique offered above, rename and define what outcomes are being sought in the learner in relation to disparity in health care outcomes. 


\section{Conclusion}

Given evidence that medical graduates can feel poorly prepared to deliver crosscultural care (Weissman, Betancourt et al. 2005), and commentary questioning the effectiveness of cultural competence curricula (Perloff, Bonder et al. 2006; Pon 2009), there is scope for new ways forward. Critically reviewing cultural competence initiatives informed by our understandings of the formal, informal and hidden curricula, provides an opportunity to reflect on what it is that educators are trying to achieve and the importance of curricular congruence. Further analysis indicates that cultural competency tends to reinforce unhelpful conceptualizations of the Other that could actually exacerbate rather than diminish health disparities (Pon 2009). The application of Hafferty's taxonomy of curricula helps to identify the intended and unintended consequences of pursuing cultural competence in health professional education. Addressing disparity in health care outcomes requires an approach to health professional education in which the formal, informal and hidden curricula are congruent. Anything less will embed cultural competence curricula as an agenda, which takes up valuable curriculum space, with little to no benefit to the populations that it is deemed to serve.

In light of this, we restate the formal curricular recommendations made earlier, including developing self-reflexivity and learning opportunities related to history and the construction of the Other, as well as communication skills training, with a focus on its complexity, rather than its simplicity. However, we also urge an approach that considers a comprehensive review of not only the formal, but also the informal and hidden curricula. Whilst a value-laden hidden curriculum often will work against the formal curriculum, changing the values of faculty provides the opportunity for reinforcement rather than undermining (Ssebunnya, 2013). Faculty professional development opportunities aimed at understanding disparity in health care outcomes 
and related health determinants for minority populations are essential. The Critical Reflection Tool, (Ewen, Mazel et al. 2012) developed in Australia and New Zealand, and adapted in Canada, provides one approach to uncovering the hidden, and to some extent the informal, curricula related to Indigenous peoples' health and development. This paper challenges faculty to consider the very language applied to the pursuit of providing the best care to all populations, arguing that the term "cultural competence" may be misleading.

If Hafferty's advice that educators should focus on what students learn rather than on what they are taught is to be followed, (Hafferty 1998) then the context and environment in which students learn requires consideration. As Taylor warns, As long as these basic features of the "hidden curriculum" and institutional culture of medical education remain in place, no amount of fine-tuning the theoretical definitions that students are assigned to read on "touchy-feely Tuesdays" is likely to unsettle the tendency of medical education to produce and reproduce itself as a "culture of no culture" (Taylor 2003 p. 558).

Mindful of a long history of reform without change in medical education (Bloom 1988) the way forward requires considering how cultural competence is expressed in the formal, informal and hidden curricula and ensuring congruence of that expression. This will provide a significant step forward in the aim of building the ability of students and graduates to be able to deliver effective care to all patients and populations. 
Acknowledgments: "The authors would like to acknowledge our colleagues involved in the Educating for Equity project. See www.educating4equity.net for more details of the project and team members."

Funding/Support: "This project is supported by funding from the National Health and Medical Research Council (Aust), grant ID 634586 and the Health Research Council (NZ), Contract number 09/643b.”

Other disclosures: "None."

Ethical approval: "Not applicable." 


\section{References}

Australian Institute of Health and Welfare (2011). Medical labour workforce 2009. AIHW Bulletin no. 89. Cat. no. AUS 138. Canberra, AIHW.

Australian Medical Council (2007). Assessment and accreditation of Medical Schools: standards and procedures. Canberra, AMC.

Bloom, S. W. (1988). "Structure and ideology in medical education: an analysis of resistance to change." Journal of Health and Social Behaviour 29(Dec): 294306.

Brooks, P. (2009). "Medical Education and the Tyranny of Competency." Perspectives in Biology and Medicine 52(1): 90-102.

Burgess, D., S. Fu, et al. (2004). "Why Do Providers Contribute to Disparities and What Can Be Done About It?" Journal of General Internal Medicine 19(11): 1154-1159.

Burson-Tolpin, A. (1989). "Fracturing the Language of Biomedicine: The Speech Play of U.S. Physicians." Medical Anthropology (New Series) 3(3): 283-293. Coory, M. and W. Walsh (2005). "Rates of percutaneous coronary interventions and bypass surgery after acute myocardial infarction in Indigenous patients " Medical Journal of Australia 182(10): 507-512.

Cross, T., B. Bazron, et al. (1989). Towards a culturally competent system of care: a monograph on the effective services for minority children who are severely 
emotionally disturbed. Washington, Georgetown University Child Development Centre.

Dogra, N., S. Reitmanova, et al. (2009). "Twelve tips for teaching diversity and embedding it in the medical curriculum." Medical Teacher 31: 990-993.

Ewen, S., O. Mazel, et al. (2012). "Exposing the Hidden Curriculum Influencing Medical Education on the Health of Indigenous People in Australia and New Zealand: The Role of the Critical Reflection Tool." Academic Medicine 87(2): 200-205.

Garvey, G., I. Rolfe, et al. (2009). "Indigenous Australian medical students' perceptions of their medical school training." Medical Education 43(11): 1047-1055.

Gregg, J. and S. Saha (2006). "Losing Culture on the Way to Competence: The Use and Misuse of Culture in Medical Education." Academic Medicine 81(6): 542547.

Hafferty, F. W. (1998). "Beyond curriculum reform: confronting medicine's hidden curriculum." Academic Medicine 73(4): 403-407.

Haider, A., J. Sexton, et al. (2011). " Association of unconscious race and social class bias with vignette-based clinical assessments by medical students." Journal of American Medical Association 306(9): 942-951.

Harris, R., B. Robson, et al. (2007). "Māori and non-Māori differences in caesarean section rates: a national review." The New Zealand Medical Journal 120(1250).

Klonoff, E. (2009). "Disparities in the provision of medical care: an outcome in search of an explanation." Journal of Behavioural Medicine 32: 48-63.

Kumagai, A. and M. Lypson (2009). "Beyond Cultural Competence: Critical Consciousness, Social Justice, and Multicultural Education." $\underline{\text { Academic }}$ Medicine 84(6): 782-787. 
Kumas-Tan, Z., B. Beagan, et al. (2007). "Measures of Cultural Competence:

Examining Hidden Assumptions." Academic Medicine 82(6): 548-557.

Liaison Committee on Medical Education (2012). Functions and structure of a medical school: Standards for accreditation of medical education programs leading to the M.D. degree. Chicago, LCME.

Merchant, J. and B. Omary (2010). "Underrepresentation of underrepresented minorities in academic medicine: the need to enhance the pipeline and the pipe." Gastroenterology 138: 19-26.

New Zealand Government (2003). Health Practitioners Competence Assurance Act 2003, New Zealand Government.

Paul, D., S. Hill, et al. (2012). "Revealing the (in)competency of 'cultural competency' in medical education." AlterNative 8(3): 318-328.

Penner, L. A., J. F. Dovidio, et al. (2010). "Aversive racism and medical interactions with black patients: a field study. ." Journal of Experimental Social Psychology 46(2): 436-440.

Perloff, R., B. Bonder, et al. (2006). "Doctor-Patient Communication, Cultural Competence, and Minority Health: Theoretical and Empirical Perspectives." American Behavioural Scientist 49(6): 835-852.

Pon, G. (2009). "Cultural Competency as new racism: an ontology of forgetting." Journal of Progressive Human Services 20(1): 59-71.

Saha, S., C. Beach, et al. (2008). "Patient Centeredness, Cultural Competence and Healthcare Quality." Journal of the National Medical Association 100(11): $1275-1285$.

Smedley, B., A. Stith, et al., Eds. (2003). Unequal treatment: confronting racial and ethnic disparities in health care. Washington, The National Academies Press. 
Ssebunnya, G. (2013). "Beyond the hidden curriculum: the challenging search for authentic values in medical ethics education." South African Journal of Bioethics and Law 6(2): 48-51.

Stern, D. (1998). "Practicing What We Preach? An Analysis of the Curriculum of Values in Medical Education." American Journal of Medicine 104: 569-575.

Taylor, J. (2003). "Confronting “Culture”, in Medicine's "Culture of No Culture”." Academic Medicine 78(6): 555-559.

Teal, C., A. Gill, et al. (2012). "Helping medical learners recognise and manage unconscious bias toward certain patient groups." Medical Education 46: 80-88. van Ryn, M. and S. Fu (2003). "Paved with good intentions: do public health and human service providers contribute to racial/ethnic disparities in health?" American Journal of Public Health 93(2): 248-255.

Wear, D., A. Kumagai, et al. (2012). "Cultural Competency 2.0: Exploring the Concept of "Difference" in Engagement With the Other." Academic Medicine 87(6).

Weissman, J., J. R. Betancourt, et al. (2005). "Resident physicians' preparedness to provide cross-cultural care." Journal of American Medical Association 294(9): 1058-1067. 


\section{University Library}

\section{- M M N E R VA A gateway to Melbourne's research publications}

Minerva Access is the Institutional Repository of The University of Melbourne

Author/s:

Paul, D;Ewen, SC;Jones, R

Title:

Cultural competence in medical education: aligning the formal, informal and hidden curricula

Date:

2014-12-01

Citation:

Paul, D., Ewen, S. C. \& Jones, R. (2014). Cultural competence in medical education: aligning the formal, informal and hidden curricula. ADVANCES IN HEALTH SCIENCES EDUCATION, 19 (5), pp.751-758. https://doi.org/10.1007/s10459-014-9497-5.

Persistent Link:

http://hdl.handle.net/11343/283177 